\title{
Investigation of room temperature protonic conduction of perovskite humidity sensors
}

\begin{abstract}
This paper shows a correlation between surface effective porosity due to various sintering regimes and humidity sensitive electrical properties of the perovskite-based bulk type humidity sensors, at room temperature. Furthermore, room temperature humidity transduction mechanism of the thick film type humidity sensors was studied in detail through electrochemical impedance spectroscopy (EIS) and major transmissive components were detected by the fitting of the Bode diagrams and Nyquist complexes to the equivalent circuits. The microstructural, morphological and elemental characterizations were carried on using XRD, EFTEM, FESEM, and EDX. Physical properties including open porosity/bulk density were investigated through American Standard Test Method (ASTM). An innovative selfdesigned material test fixture with ceramic supports was fabricated for a high $\mathrm{S} / \mathrm{N}$ ratio electrical measurement of the bulk samples. All the sensors were set up at 20-95\% RH. The morphological, physical, and electrical results of the bulk pellets indicate a direct correlation of the open cavities and AC conduction. The higher the open porosity is, the greater is the conduction and vice versa. Presence of the ionic transport is clearly observed from the frequency-conductance spectra at room temperature. Noise-free detected behavior via EIS proves that the proton transfer mechanism is a dominant responsible, and executed by both charge transfer resistance and kinetically controlled charge transfer (diffusive species) at low and middle to high RH. Next to the Warburg effect (at $80 \% \mathrm{RH}$ ), for the first time, a Gerischer impedance was found as a dominant agent of transduction at $85 \% \mathrm{RH}$ to above.
\end{abstract}

Keyword: Gerischer element; Humidity sensitive; Ionic diffusion; Nanocomposite; Perovskite; Thick film; Warburg 